\title{
LUT
}

University

\section{Novel Smart and Compliant Robotic Gripper: Design, Modelling, Experiments} and Control

\author{
Milojevic Andrija, Tomic Misa, Handroos Heikki, Cojbasic Zarko
}

This is a Final draft

version of a publication

published by IEEE

in IEEE EUROCON 2019 -18th International Conference on Smart Technologies

DOI: $10.1109 / E U R O C O N .2019 .8861561$

Copyright of the original publication: (C) 2019 IEEE

Please cite the publication as follows:

A. Milojević, M. Tomić, H. Handroos and Ž. Ćojbašić, "Novel Smart and Compliant Robotic

Gripper: Design, Modelling, Experiments and Control," IEEE EUROCON 2019 -18th International Conference on Smart Technologies, Novi Sad, Serbia, 2019, pp. 1-6, doi: 10.1109/

EUROCON.2019.8861561.

(c) 2019 IEEE. Personal use of this material is permitted. Permission from IEEE must be obtained for all other uses, in any current or future media, including reprinting/republishing this material for advertising or promotional purposes, creating new collective works, for resale or redistribution to servers or lists, or reuse of any copyrighted component of this work in other works.

This is a parallel published version of an original publication. This version can differ from the original published article. 


\section{Novel Smart and Compliant Robotic Gripper: Design, Modelling, Experiments and Control}

\author{
Andrija Milojević \\ Department of \\ Mechanical Engineering \\ Lappeenranta University of Technology \\ Lappeenranta, Finland \\ Andrija.Milojevic@lut.fi \\ Heikki Handroos \\ IEEE Member \\ Department of Mechanical Engineering \\ Lappeenranta University of Technology \\ Lappeenranta, Finland \\ heikki.handroos@lut.fi
}

\author{
Miša Tomić \\ Mechanical \\ Engineering Faculty \\ University of Niš \\ Niš, Serbia \\ misa.tomic@masfak.ni.ac.rs \\ Žarko Ćojbašić \\ Senior IEEE Member \\ Mechanical Engineering Faculty \\ University of Niš \\ $\mathrm{Niš,} \mathrm{Serbia}$ \\ zcojba@ni.ac.rs
}

\begin{abstract}
This paper presents a further investigation of a novel adaptive soft robotic gripper with integrated active elements, i.e. sensors and actuators. In many contemporary robotic applications, there is a great need to grasp differently shaped and sized objects as well as objects of different stiffness or soft objects. Grippers based on classical rigid-body mechanisms cannot safely manipulate with such a wide variety of objects, as they are stiff and not adaptive. Adaptive gripper proposed here is based on elastic active compliant mechanisms. Via integrated sensors, and actuators active smart gripper changes the shape of its grasping surface and adapts to the object of unknown shape. Concept of a two-finger gripper and a functional prototype of one gripper finger are presented. Sensors are formed by using conductive graphite foam. The machine learning models of tactile foam sensors are presented. Actuators are formed by using a shape memory alloy wire nitinol. It is demonstrated that developed soft gripper concept with integrated active elements can realize multiple grasping patterns. Many potential applications in robotics are foreseen for the novel smart adaptive gripper.
\end{abstract}

Keywords—robotics, adaptive gripper, compliant mechanisms, conductive foam sensors, nitinol wire sensors

\section{INTRODUCTION}

Handling of objects of various shapes and sizes, as well as handling of fragile and soft objects, is required in many contemporary robotics applications. For safe grasping and manipulation of such a wide variety of objects, adaptability is required. Grippers that utilize rigid-body mechanisms cannot realize the needed level of adaptability and they generally cannot handle well soft and fragile objects. Developing an adaptive compliant gripper that can realize manipulations of a wide variety of objects, represents a challenging task. Adaptive smart gripper solution could respond to external stimuli during grasping and could realize shape morphing of its grasping surface and achieve appropriate adaptation to objects of various shapes. Applications in different industries would benefit from such kind of gripper. Moreover, industrial robots with adaptive soft gripper would be flexible and adaptive to their working environment.

In recent years various kinds of soft robotic grippers that achieve some measure of adaptability were developed [1-10]. Most of the developed grippers have disadvantages like: need for complex assembling [1,8], bulky external drives (motors, compressed air) [1, 3-5, 7-10], high operating voltage [6], limited possibility for scaling, complicated and expensive solutions, no unique synthesis methodology how to design adaptive gripper.

This paper presents a novel concept of the adaptive soft robotic gripper. One way to develop adaptive shape morphing gripper is to utilize active compliant systems i.e. compliant mechanisms with integrated sensors and actuators (Fig. 1). Flexible monolithic structures that utilize elastic deformation of its structural segments, to realize force and motion transmission, are usually known as compliant mechanisms [11]. Such mechanisms offer different benefits over rigidbody mechanisms: reduce complexity, easy to manufacture, no assembly, friction-free motion, better scalability. Compliant mechanisms deform as one whole structure, thus providing one unique advantage for achieving shape morphing and adaptability. By integrating sensors and actuators within the compliant mechanism structure, it is possible to develop a system that can realize controllable shape morphing. This kind of compliant system can be utilized to realize soft robotic gripper. By using integrated actuation and via compliant structure, the gripper can realize controllable shape morphing of its grasping surface i.e. can realize multiple grasping patterns thus achieving structural adaptability. Information from the integrated sensors in the gripper system can be used to detect contact with the objects. This information can also provide feedback to realize appropriate control of actuators and grasping surface and achieve needed shape morphing. Such gripper would be able to adapt to the unknown shaped objects (Fig. 1).

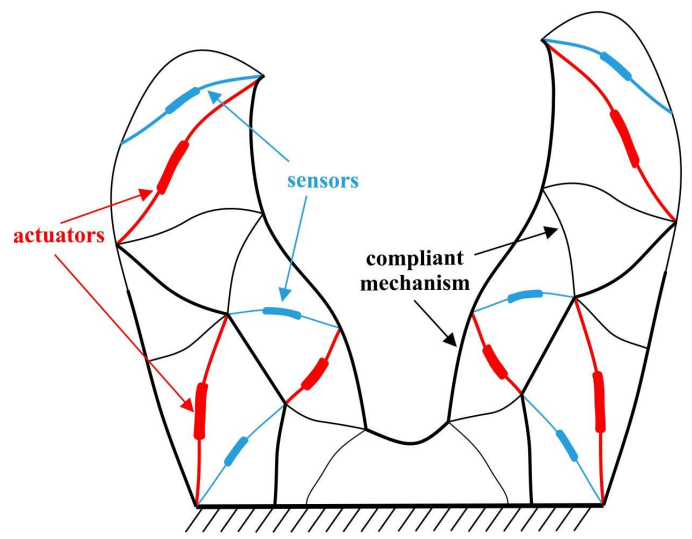

Fig. 1. Illustration of a compliant system (compliant mechanisms with integrated sensors and actuators) as an adaptive soft robotic gripper. 
The concept design of the full two-finger gripper is already presented in our previous paper [12]. The prototype model of one finger gripper with integrated smart material actuators and sensor was introduced as well in [12]. Actuators were formed by using shape memory alloy wires (SMA) [13]. Sensors were formed by using conductive foam material [12]. The simple control algorithm was developed as well. It was shown that such griper concept can realize controllable shape morphing of its grasping surface via SMA actuators and when conductive foam sensors are used to detect pressure. But detailed investigations regarding sensor behaver and model were not introduced.

Capabilities and adaptability of a gripper have been further investigated in this paper by using geometrically nonlinear FEM. It was shown that the obtained solution of the gripper could realize different grasping patterns but when only contracting actuators are used, while other possibilities were not investigated. In this paper, further analysis and results are presented when a combination of contracting and expanding actuators are used. This paper presents also the new model for prediction of conductive graphite foam-based sensor behavior when they are used as displacement sensors. The model is developed by using machine learning. The integration of sensors with the gripper finger prototype is presented. Possibilities to use the developed sensor model and information derived from them to realize smart gripper concept i.e. more advanced control of gripper grasping surface are described at the end.

\section{Design Methodology For AdAPtiVE SOFT ROBOTIC GRIPPER}

The synthesis methodology for the adaptive soft robotic gripper is already presented in $[12,14]$. Here only a very brief summary is given.

To develop the adaptive robotic gripper, compliant systems i.e. compliant mechanisms with integrated actuators are used [11]. Synthesis method for such systems includes: defining the problem specifications (Fig. 2a); parameterization of the design domain [14] (Fig. 2b); and application of genetic algorithms [15] as an optimization method for finding the optimal solution (Fig. 2c).

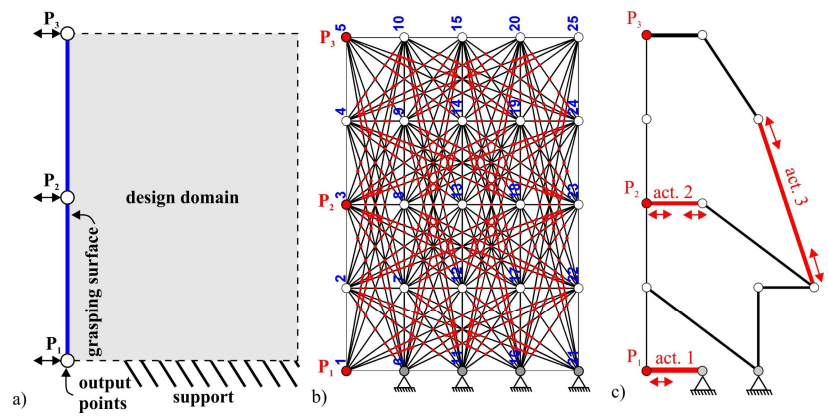

Fig. 2. The synthesis of the adaptive soft robotic gripper finger: a) problem specifications; b) design domain parameterization; c) optimal solution.

In order to simplify the optimization process, the synthesis of only one finger of the gripper is done. Based on this 2, 3 or multiple finger gripper can be easily designed. Optimization approach includes [14] the simultaneous synthesis of mechanism structural topology and actuator placement (orientation, location, size). Here the forming of mechanism structure affects how the actuators are placed in the structure and vice versa. The variables that are optimized are the beam thickness (structural elements of the mechanism) and a variable that selects actuators in the structure [14]. Here the beams can have different thickness and can become an actuator. The goal of optimization is to find optimal topology i.e. optimal outline of the compliant structure with integrated actuators so that system achieves maximal structural controllability (adaptability) [14] of its grasping surface.

The obtained optimal solution of adaptive soft robotic gripper finger is shown in Fig. 2c.

\section{Two-FINGER ADAPTIVE SOFT RoBOtIC GRIPPER DESING}

By using the obtained optimal solution (Fig. 2c) a prototype model of the two-finger gripper concept is designed (Fig. 3). Here, the actuators are modeled in the form of thin elastic spring elements (" $\mathrm{S}$ " form) first.

The actuators are modeled in such a way, in order to simulate the general behavior of any actuator that can contract/expand i.e. to show the behavior of the gripper fingers when actuators contract/expand. To investigate the behavior of the two-finger gripper, a nonlinear FEM simulation is performed by using commercially available FEM software. For setting up the FEM simulations, the following initial conditions are set:

- fixed bounded condition is applied to the bottom surface of the gripper frame (Fig. 3),

- input displacement of $5 \mathrm{~mm}$ is applied at both ends of all the actuators (total stroke of actuators is $10 \mathrm{~mm}$, black arrows); to realize shape morphing of the grasping surface,

- to realize closing of the gripper, input displacement of $5 \mathrm{~mm}$ is also applied at the input port of the gripper (red arrows).

Fig. 4 shows the behavior of two-finger gripper when contracting actuator 1 and expanding actuator $1 \mathrm{a}$ (Fig. 4a), contracting act. 2 and expanding act. 2a (Fig. 4b) or contracting act. 3 and expanding act. 3a (Fig. 4c) are active. Different irregular shaped objects can be grasped when a combination of contracting and expanding actuators is used.
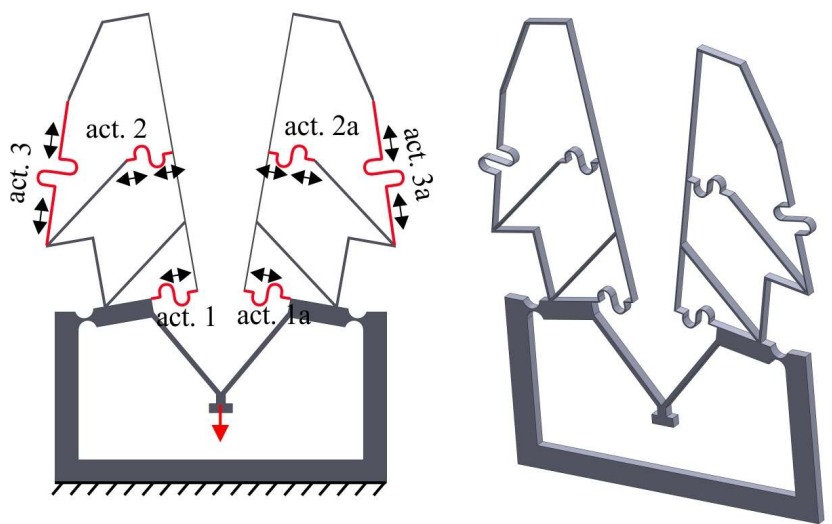

Fig. 3. The concept of the adaptive soft robotic two-finger gripper.

For example, when actuators 2 and $2 \mathrm{a}$ are active or actuators 3 and $3 \mathrm{a}$, a convex-concave shaped object can be grasped (Fig. 4b and Fig. 4c) and when actuator 1 and 1a are active an object with additional straights can be grasped (Fig. 4a). More grasping patterns could be realized if multiple actuators at one gripper finger are active at the same time. Concave and convex objects of different size could be grasped 
for different input displacement at the input port of a gripper as well as for different strokes of the actuators. More variety of different objects could be grasped if only extending or a different combination of contracting and extending actuators are used too (than the ones explored in this paper).

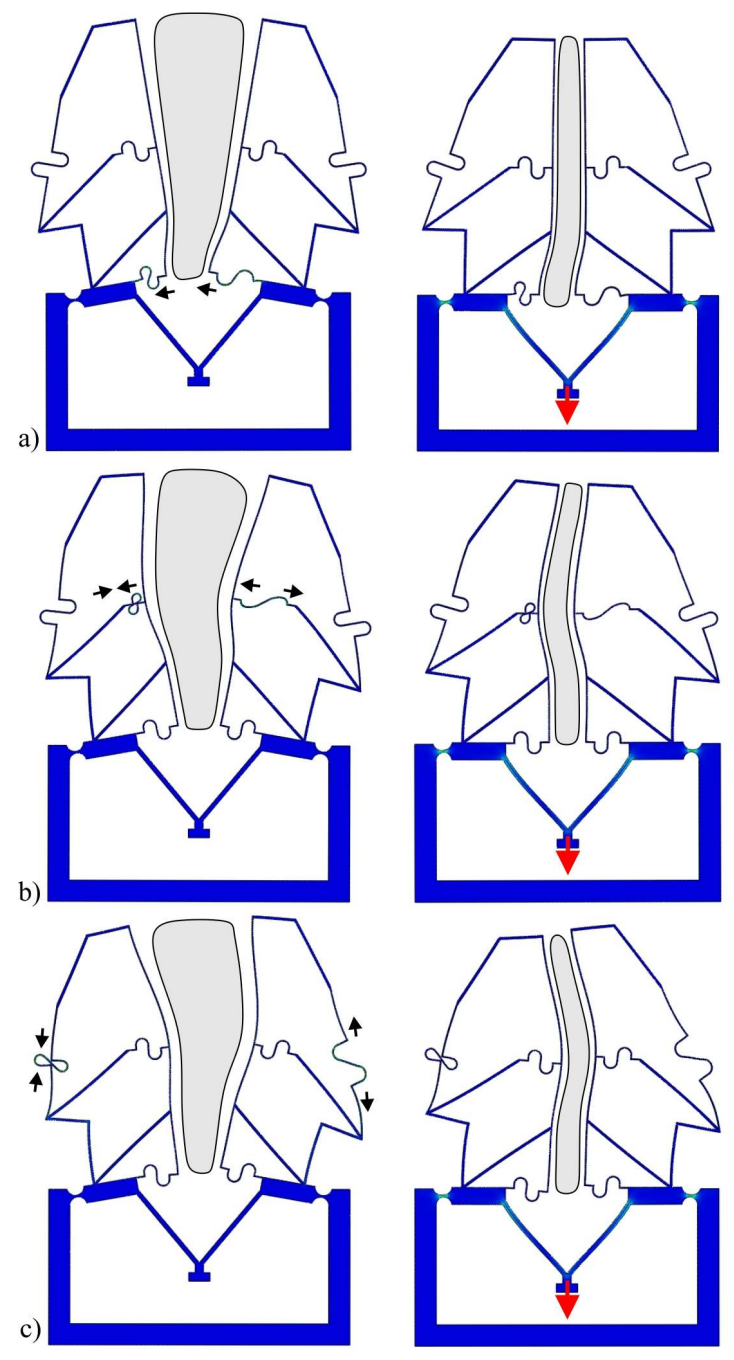

Fig. 4. FEM results of the two-finger gripper behavior when contracting and expanding 1 and $1 \mathrm{a}(\mathrm{a}), 2$ and $2 \mathrm{a}$ (b) or 3 and $3 \mathrm{a}$ (c) are active (in the figures to the right gripper fingers are shown in closed-end positions).

The results of FEM simulations show that the adaptive two-finger gripper can realize different grasping patterns and has the potential to adapt to different shaped objects.

\section{ACTUATORS}

Real actuators need to be used in order to realize controllable shape morphing of the gripper grasping surface.

Classical actuators such as electro-motors, hydraulic and pneumatic cylinders, are considered to have infinite stiffens. Thus, they can not be used as actuators of compliant systems. Many researchers developed different kind of actuators that are suitable for application in compliant systems, such as artificial muscles $[16,17]$, piezoelectric actuators, compliant fluidic actuators, smart polymers, and shape memory alloys (SMA) [17].

Among all the actuators, SMA was chosen as the most suitable for application in the developed gripper. SMA represents a material when heated above a certain temperature (activation temperature), and if previously deformed and distorted, it can retain its "memorized" pre-deformed shape $[13,18]$. This property of SMA (to return to its memorized shape) can be used for actuation. By using SMA in the form of a wire it is possible to heat-treat the wire ("train" the wire) to remember the particular shape. Thus, it is possible to develop different kinds of actuators.

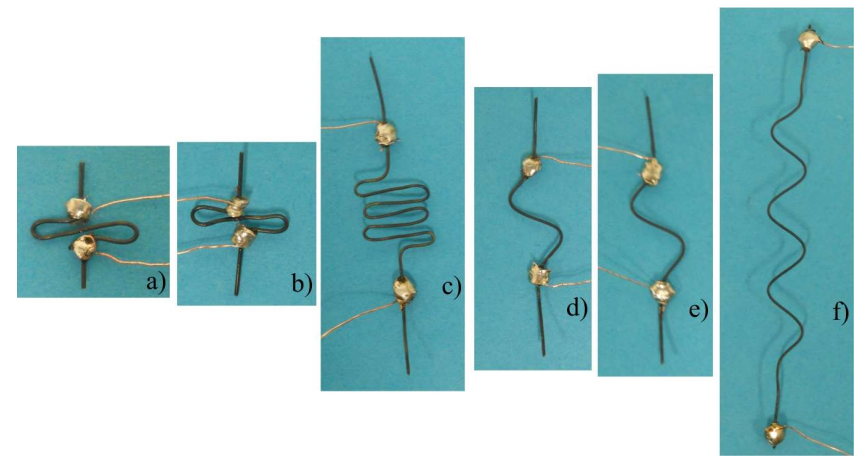

Fig. 5. Nitinol wire actuators: a) actuator 1 ; b) actuator 2 ; c) actuator 3; d) actuator $1 b$; e) actuator $2 b$; f) actuator $3 b$.

In [12], details explanation is given about forming the SMA actuators for the developed soft robotic gripper. Here the final form of actuators is presented. Fig. 5 shows the contacting and expanding SMA actuators that are used as actuators of robotic gripper finger.

First contracting SMA actuators are integrated within the compliant structure (Fig. 6a to Fig. 6c). After the activation of contracting actuators, gripper finger will stay in its deformed position (Fig. 6d to Fig. 6f), as nitinol wires cannot realize repetitive motion and return the grasping surface in its initial position. To realize repetitive motion i.e. active stroke of the actuators in both directions, an additional set of extending actuators is used in a parallel plane (at the same place like their corresponding contracting actuators).

Fig. $6 \mathrm{~g}$ to Fig. $6 \mathrm{i}$ shows the behavior of gripper finger when individual contracting SMA actuators are activated, while both contracting and expanding actuators are integrated. The contacting actuators for cases shown in Fig. 6d to Fig. 6f, are activated by applying the voltage of $1.5 \mathrm{~V}$. For cases shown in Fig. $6 \mathrm{~g}$ to Fig. 6i, all the actuators are activated by applying the voltage of $3 \mathrm{~V}$.

To realize return stroke of the actuators 1,2 and 3 i.e. to return the grasping surface in its initial (undeformed) shape, extending actuators $(1 \mathrm{~b}, 2 \mathrm{~b}, 3 \mathrm{~b})$ are activated. By analyzing the behavior of the compliant system in Fig. $6 \mathrm{~g}$, it is observed that all three actuators $(1 \mathrm{~b}, 2 \mathrm{~b}$, and $3 \mathrm{~b})$ return the grasping surface in the initial shape. In this way, adaptive shape morphing gripper finger can realize controllable displacement in both directions. It could be concluded that the developed adaptive soft robotic gripper finger with integrated contracting and extending actuators could realize controllable shape morphing and thus multiple shapes (grasping patterns) of its grasping surface.

\section{SENSORS}

The tactile sensors in a form of soft or artificial robotic skin are suitable for application in the developed robotic gripper. Different type of tactile sensors was developed by others [19 - 20]. But most of these sensor solutions are complicated to realize and expensive. Thus, here different concept of a simple, low-cost sensor is developed by utilizing conductive polyurethane graphite foam (Fig. 7). 

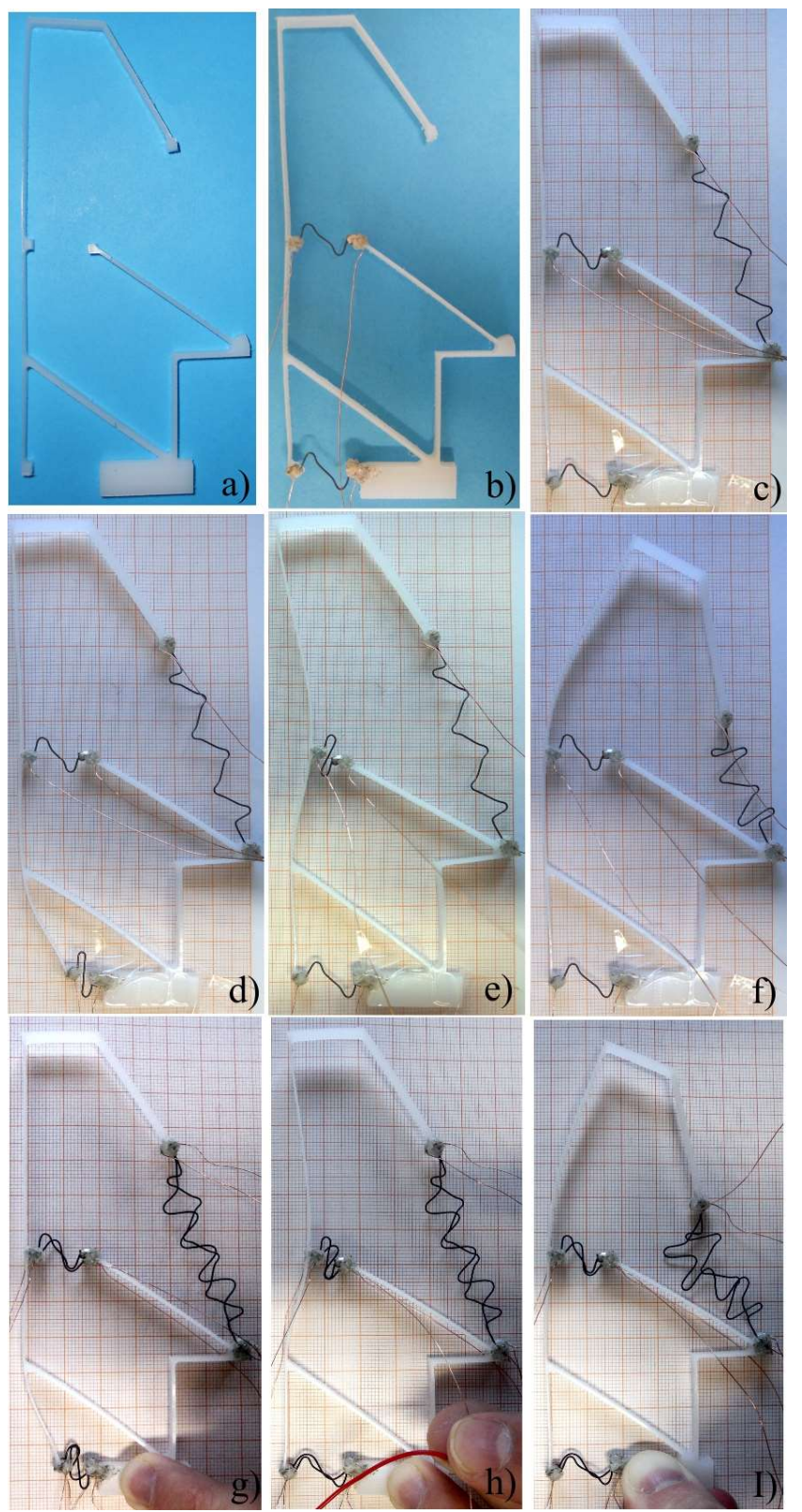

Fig. 6. Adaptive gripper finger with integrated nitinol wire actuators: (a) gripper finger structure, (b) integration of contracting actuators 1 and 2, (c) integration of contracting actuator 3 , and behavior when contracting actuator 1 (d), 2 (e), and 3 (f) is active and behavior when expanding actuators are integrated while contracting actuators $1(\mathrm{~g}), 2$ (h) and 3 (i) are active.

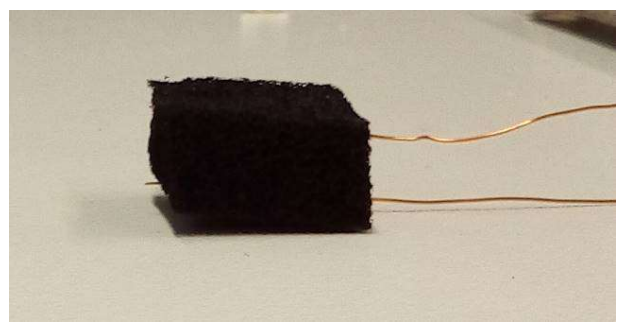

Fig. 7. Conductive graphite foam-based sensor.

Displacement sensors using conductive graphite foam can be formed by inserting two copper wires into the graphite foam (Fig. 7). Wires are parallel to each other and placed at the opposite ends of the foam piece. It is important to ensure that there is no direct electric contact between two copper wires. Thus, it is recommended to use enameled copper wires, which ends are cleaned only on the length inside the conductive foam.
When pressure is applied on the foam, graphite particles inside the foam become closer and make better electric contact, which leads to the decrease of the electric resistance. Also, when the foam is released, its electric resistance increases, because the graphite particles inside the foam become more separated (distanced from each other). By using a Wheatstone bridge, changes in electrical resistance can be easily transformed into voltage changes.

The main problem of conductive foam-based displacement sensors is that the displacement-electrical resistance characteristic, of conductive foam, is highly nonlinear and with hysteresis. These problems have been overcome by developing the model of the conductive graphite foam displacement sensors by utilizing machine learning, i.e. the artificial neural network (ANN) [22][23].

In order to provide the data for training of the ANN, an experiment was done, where a specimen of the conductive graphite foam with dimensions $15 \times 15 \times 8 \mathrm{~mm}$ was cyclically pressed and released (10 cycles) while displacement was measured by inductive sensor (Fig. 8). The pressure force is applied in the direction as shown in Fig. 8c. The data of the displacement obtained by the inductive sensor, as well as the data of voltage changes of the conductive graphite foam (Fig. 9) from Wheatstone bridge were stored and later used for training the ANN. A standard feed-forward, back propagation $\mathrm{ANN}$ is used to determine the displacement of the graphite conductive foam displacement sensor when pressure is applied to the sensor, based on measured voltage change in Wheatstone bridge due to electric resistance change. To provide an efficient solution, the artificial neural network has three layers: an input layer, a single hidden layer and an output layer (Fig. 10). As the network input variable, the voltage change is selected, while the selected output network variable is displacement obtained by the inductive sensor.

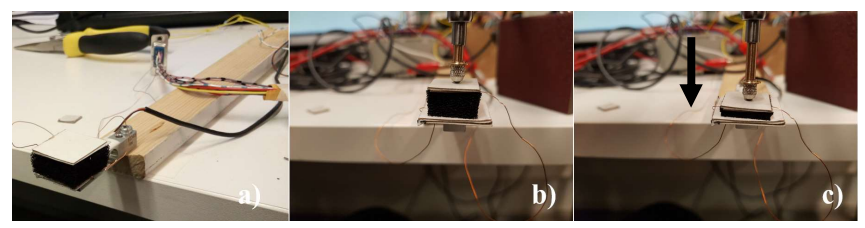

Fig. 8. Experimental setup (a) with graphite foam-based sensor shown in undeformed (b) and deformed position (c).

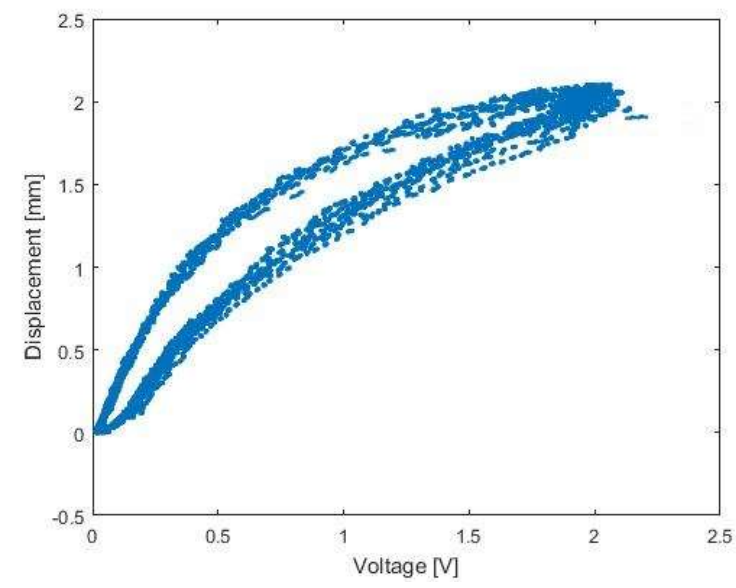

Fig. 9. Measured results when conductive graphite foam is cyclically pressed - graphite foam displacement with respect to the foam voltage change.

The developed ANN model was tested for the conductive foam displacement sensors made from other conductive foam 
pieces then ones used for obtaining the data for training the ANN but formed on the same way and with same dimensions. The test was performed by obtaining data from new conductive foam displacement sensor using data acquisition devise NI USB 6009, sending this data into the ANN as input and comparing the output data from the ANN with the data obtained by the inductive displacement sensor.

Fig. 11 shows signals from graphite conductive foam displacement sensor transformed using developed ANN model (white color), and inductive displacement sensor (red color), and it shows that there is good matching between graphite conductive foam displacement sensor and inductive displacement sensor.

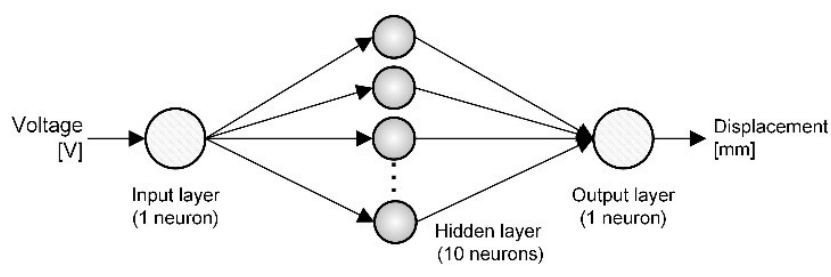

Fig. 10. An artificial neural network with 1 input variable, 1 hidden layer with 10 neurons, and 1 output variable.

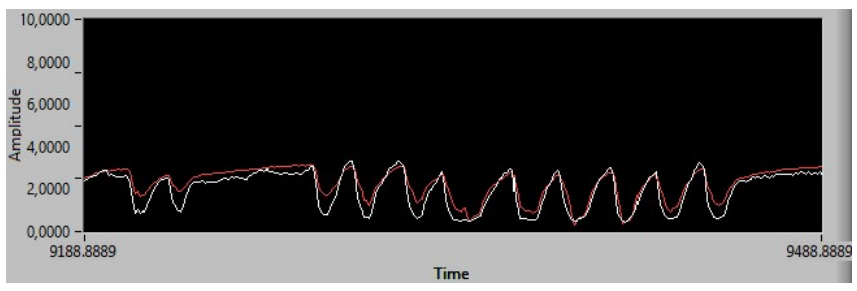

Fig. 11. ANN conductive foam displacement sensors model test.

\section{ADAPTIVE SOFT ROBOTIC GRIPPER FINGER WITH INTEGRATED ACTUATORS AND SENSORS}

To develop an adaptive smart gripper finger that can detect contact with its environment, conductive foam is integrated across the whole shape morphing or grasping surface (Fig. 12a).

Two sets of thin copper wires are integrated at six different locations within the conductive foam to realize six sensors (Fig. 12a). This means that gripper finger can detect pressure and via the developed sensor model, measure displacement at six different points at the grasping surface. The gripper finger can detect pressure/measure displacement at any point on the grasping surface if the sensor is formed at the desired location of detection (by inserting two parallel copper wires).

Fig. 12b shows the corresponding virtual instrumentation built in LabView for measuring displacement and displaying the results when pressure is applied to all six points respectively. Simple control algorithm (Fig. 14) is developed here in order to show the possibilities of the developed gripper finger. The control algorithm is such that individual sensors on the grasping surface activate corresponding nitinol actuators.

Fig. 13 shows the behavior of the adaptive gripper finger when pressure is applied to the grasping surface. Every sensor is pressed separately to activate the corresponding actuator.

By measuring the displacement from the sensors when gripper comes into contact with objects of unknown shape, it is possible to develop much more complex control algorithm that would provide gripper with ability to realize appropriate shape morphing i.e. grasping pattern.
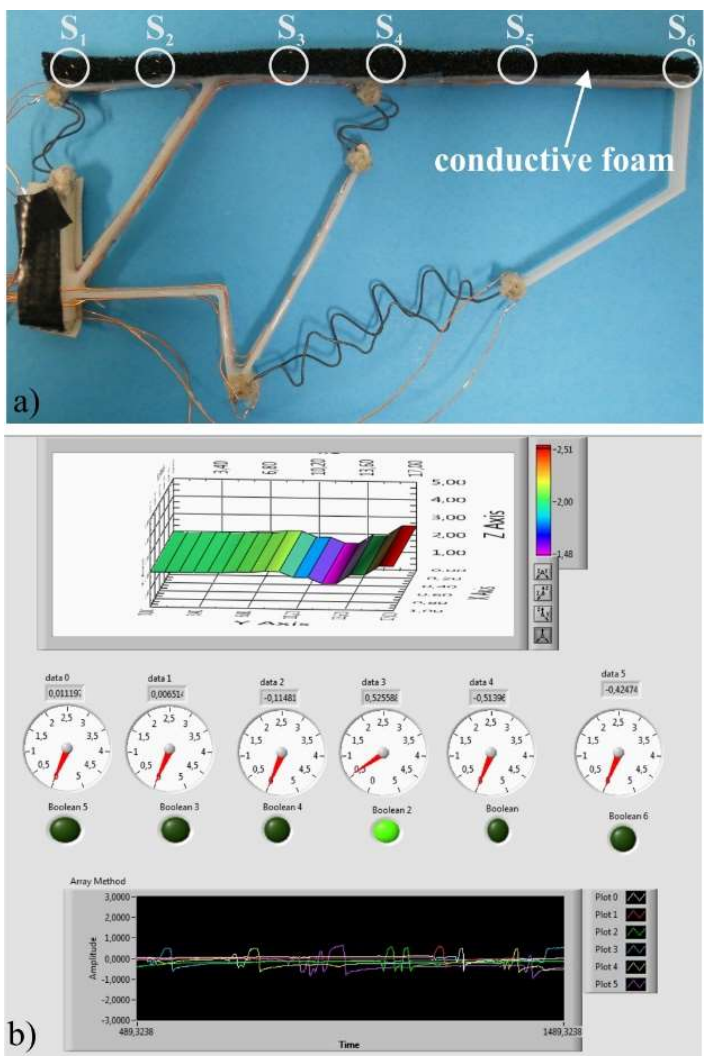

Fig. 12. a) Conducive foam integration in gripper finger and forming of sensors; b) virtual instrumentation for foam sensors behavior tracking.
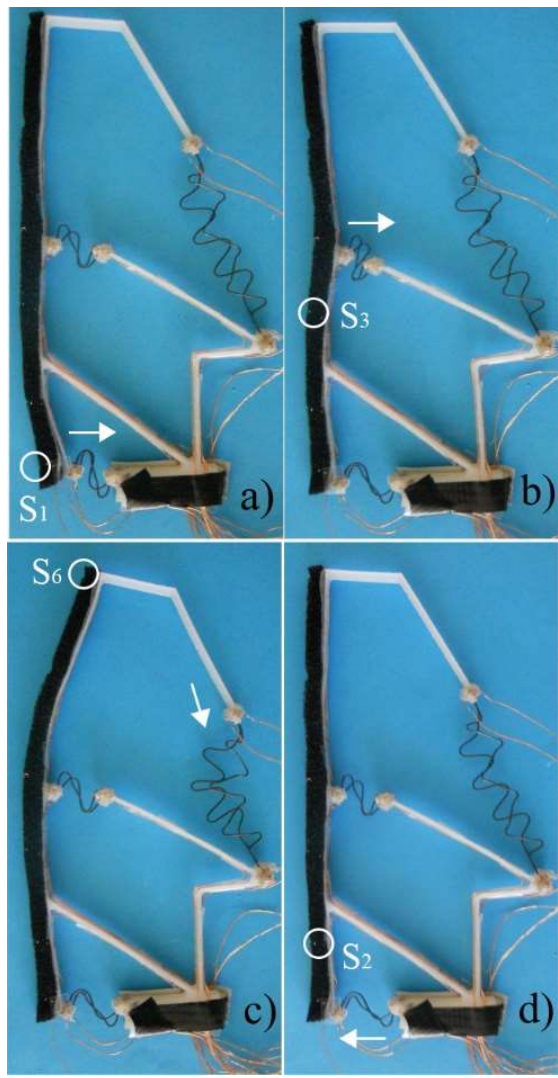

Fig. 13. Behavior of gripper finger with integrated nitinol actuators, foam sensors and control algorithm: a) activation of contracting actuator 1 by detecting contact in point $\mathrm{S} 1 ; \mathrm{b}$ ) activation of actuator 2 by detecting contact in point $\mathrm{S} 3$; c) activation of actuator 3 by detecting contact in point $\mathrm{S} 6$; d) activation of expanding actuator $1 \mathrm{~b}$, by detecting contact in point $\mathrm{S} 2$. 


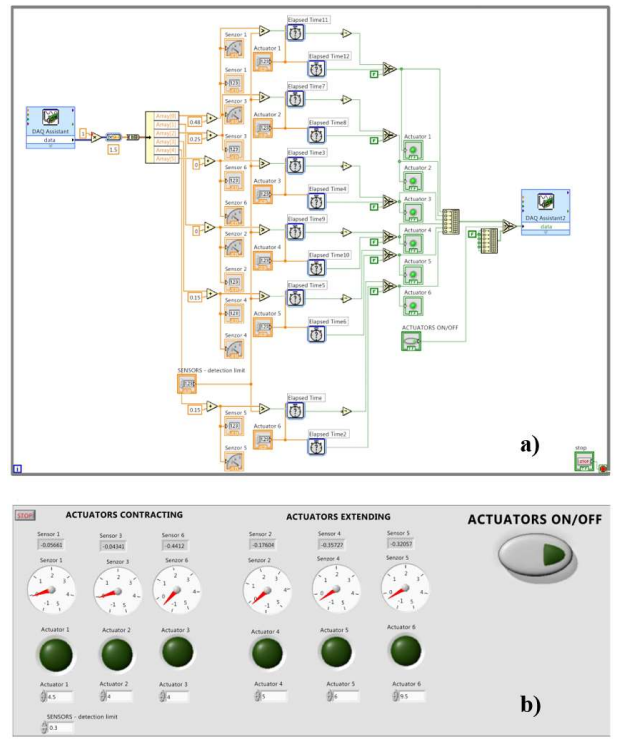

Fig. 14. a) Control algorithm for adaptive gripper finger with integrated nitinol actuators and conductive foam-based sensors; $b$ ) corresponding virtual instrumentation.

The results show that the developed adaptive gripper finger with integrated nitinol wire actuators and conductive foam sensors can realize controllable shape morphing, i.e. multiple shapes of its grasping surface, autonomously adapting according to the implemented control algorithm.

\section{CONCLUSION}

In this paper, the novel smart active and compliant robotic gripper is presented. Results including design, modeling, experimental investigations and control are shown.

The concept of the two-finger gripper is introduced and FEM results of gripper behavior are presented. Further, it is demonstrated that by integrating the SMA nitinol wire actuators within the compliant structure, it is possible to develop a gripper that can achieve multiple shapes of its grasping surface, i.e. system gains the property of structural adaptability. By integrating sensors formed of conductive foam the gripper can detect contacts with objects. Also, by using an appropriate control algorithm gripper system based on the signals from the sensors can decide which actuator to activate to achieve appropriate shape morphing and adapt to the unknown objects.

In this paper, simple on/off control of the SMA actuators is presented, while controlling the stroke of the actuators could be explored in the future. The functional model of one gripper finger was introduced, while the full realization of two-finger gripper porotype, object grasping and a wider range of possible applications remain to be further investigated in future work.

\section{ACKNOWLEDGMENT}

The research was supported by The Strategic Research Council (SRC) at the Academy of Finland, Serbian Academy of Sciences and Arts (SMSS project) and Academy of Finland Research Council for Natural Sciences and Engineering (No. 318390). The financial support is gratefully acknowledged.

\section{REFERENCES}

[1] H. Choi and M. Koc, "Design and feasibility tests of a flexible gripper based on inflatable rubber pockets," Int. J. Mach. Tools Manu., vol. 46, no. $12-13$, pp. 1350-1361, Oct. 2006.
[2] G.-P. Jung, J.-S. Koh, and K.-J. Cho, "Underactuated adaptive gripper using flexural buckling," IEEE Trans. Robotics, vol. 29, no. 6, pp. 1396-1407, Dec. 2013.

[3] M. E. Giannaccini, I. Georgilas, I. Horsfield, B. H. P. M. Peiris, A. Lenz, A. G. Pipe, and S. Dogramadzi, "A variable compliance, soft gripper," Auton. Robot., vol. 36, no. 1-2, pp. 93-107, Jan. 2014.

[4] R. V. Martinez, J. L. Branch, C. R. Fish, L. Jin, R. F. Shepherd, R. M. D. Nunes, Z. Suo, and G. M. Whitesides, "Robotic tentacles with threedimensional mobility based on flexible elastomers," Adv. Mater., vol. 25, no. 2, pp. 205-212, Jan. 2013.

[5] F. Ilievski, A. D. Mazzeo, R. F. Shepherd, X. Chen, and G. M. Whitesides, "Soft robotics for chemists," Angew. Chem. Int. Ed., vol. 50, no. 8, pp. 1890-1895, Feb. 2011.

[6] O. A. Araromi, I. Gavrilovich, J. Shintake, S. Rosset, M. Richard, V. Gass, and H. R. Shea, "Roll-able multisegment dielectric elastomer minimum energy structures for a deployable microsat-ellite gripper," IEEE/ASME Trans. Mech., vol. 20, no. 1, pp. 438-446, June 2014.

[7] J. R. Amend, E. Brown, N. Rodenberg, H. Jaeger, and H. Lipson, “A positive pressure universal gripper based on the jamming of granular material," IEEE Trans. Robot., vol. 28, no.2, pp. 341-350, Jan. 2012.

[8] R. A. J. Stavenuiter, L. Birglen, and J. L. Herder, "A planar underactuated grasper with adjustable compliance," Mechanism and Machine Theory, vol. 112, pp. 295-306, June 2017.

[9] K. C. Galloway, K. P. Becker, B. Phillips, J. Kirby, S. Licht, D. Tchernov, R. J. Wood, and D. F. Gruber, "Soft Robotic Grippers for Biological Sampling on Deep Reefs," Soft Robotics, vol. 3, no. 1, pp. 23-33, Jan. 2016.

[10] S. Terryn, J. Brancart, D. Lefeber, G. van Assche, and B. Vanderborght, "Self-healing soft pneumatic robots," Science Robotics, vol. 2, no. 9, eaan4268 (12pp), Aug. 2017.

[11] L. L. Howell, S. P. Magleby, and B. M. Olsen, Handbook of Compliant Mechanisms. Chichester: John Wiley \& Sons, 2013.

[12] A. Milojevic, S. Lin $\beta$, Z. Cojbasic, H. Handroos, L. Luostarinen, and L. Zentner, "Adaptive Soft Robotic Gripper Based on Shape Morphing Compliant System," in Proc. 2018 International Conference on Reconfigurable Mechanisms and Robots (ReMAR), Delft, 2018, 10.1109/REMAR.2018.84498311 pp. 1-10.

[13] J. M. Jani, M. Leary, A. Subic, and M. A. Gibson, "A review of shape memory alloy research, applications and opportunities," Mater. Des., vol. 56, pp. 1078- 1113, April 2014.

[14] A. Milojević and N. D. Pavlović, "Development of a new adaptive shape morphing compliant structure with embedded actuators," J. Int. Mater. Syst. Struct., vol. 27, no. 10, pp. 1306-1328, June 2016.

[15] R. L. Haupt and S. E. Haupt, Practical Genetic Algorithms. New Jersey: John Wiley \& Sons, 2004.

[16] G. Kovacs, L. Düring, S. Michel, and G.Terrasi, "Stacked dielectric elastomer actuator for tensile force transmission," Sensors and Actuators A: Physical, vol. 155, no. 2, pp. 299-307, Oct. 2009.

[17] G. Berselli, R. Vertechy, and G. Vassura, Smart Actuation and Sensing Systems - Recent Advances and Future Challenges. Rijeka: InTech Publisher, 2012.

[18] J. J. Mohd, M. Leary, and A. Subic, "Designing shape memory alloy linear actuators: A review,” J. Int. Mater. Syst. Struct., vol. 28, no. 13, pp. 1699-1718, Dec. 2016.

[19] Park Y.-L., Chen B.-R., and Wood R. J., "Design and Fabrication of Soft Artificial Skin Using Embedded Microchannels and Liquid Conductors,” IEEE Sens. J., vol. 12, no. 8, pp. 2711-2718. Aug. 2012.

[20] H. Tian, Y. Shu, X.-F. Wang, M. A. Mohammad, Z. Bie, Q.-Y. Xie, C. Li, W.-T. Mi, Y. Yang, and T.-L. Ren, "A Graphene-Based Resistive Pressure Sensor with Record-High Sensitivity in a Wide Pressure Range," Scientific Reports, vol. 5, 8603 (6pp.). Feb. 2015.

[21] S. Brady, D. Diamond, and K.-T. Lau, "Inherently conducting polymer modified polyurethane smart foam for pressure sensing," Sensors and Actuators A: Physical, vol. 119, no. 2, pp. 398-404. April 2005.

[22] Ž. Ćojbašić, V. Nikolić, E. Petrović, V. Pavlović, M. Tomić, I. Pavlović, I. Ćirić, "A Real Time Neural Network Based Finite Element Analysis of Shell Structure", Facta Universitatis, Series: Mechanical Engineering, Vol. 12, No 2, pp. 149 - 155, 2014.

[23] D. Petković, N. D. Pavlović, Ž. Ćojbašić, N. T. Pavlović (2013), “Adaptive neuro fuzzy estimation of underactuated robotic gripper contact forces", Expert Systems With Applications, Volume 40, Issue 11, pp. 281-286, DOI: 10.1016/j.eswa.2012.07.076, January 2013. 